\title{
Editorial
}

\section{Smart Technologies for Vehicle Safety and Driver Assistance}

\author{
Mihai Dimian (D), ${ }^{1}$ Luc Chassagne, ${ }^{2,3}$ Petru Andrei, ${ }^{4}$ and $\mathrm{Pu} \mathrm{Li}^{5}$ \\ ${ }^{1}$ Stefan cel Mare University of Suceava, Suceava 720229, Romania \\ ${ }^{2}$ University of Paris-Saclay, Saint-Aubin, France \\ ${ }^{3}$ University of Versailles Saint-Quentin-en-Yvelines, Vélizy-Villacoublay 78140, France \\ ${ }^{4}$ Florida State University, Florida A\&M University, Tallahassee, Florida 32310, USA \\ ${ }^{5}$ Technical University of Ilmenau, Ilmenau 98693, Germany \\ Correspondence should be addressed to Mihai Dimian; dimian@usm.ro
}

Received 16 April 2019; Accepted 16 April 2019; Published 15 May 2019

Copyright (C) 2019 Mihai Dimian et al. This is an open access article distributed under the Creative Commons Attribution License, which permits unrestricted use, distribution, and reproduction in any medium, provided the original work is properly cited.

Transportation is currently facing significant challenges from a variety of factors including growing populations and number of vehicles, rising urbanization, negligent driving, and environmental issues. An interdisciplinary approach is needed to address these complex problems, and information and communications technologies (ICT) are emerging as leading contributors to the future of transport. With more than one million victims in road accidents annually, the improvement in safety and efficiency of the transportation system is of major interest for industrial and academic research. The use of ICT in development of intelligent safety systems, driver assistance systems, cooperative driving networks, and smart transport infrastructure is expected to contribute to significant reduction of road accident and traffic congestion. In this context, this special issue aims at providing a collection of innovative and high-quality research contributions focusing on smart technologies for vehicle safety and driver assistance. 28 papers were submitted for this issue; 12 of them were accepted for publication and were summarized below.

The paper by P. Das et al. demonstrates a proof of concept of simulation-based framework for evaluating safety performances of Intelligent Transportation System (ITS) countermeasures. The traffic microsimulation model and surrogate safety assessment model are used to estimate crash modification factors while a case study is used to calibrate the model to real-world scenario. This approach can investigate the combined effect of multiple proposed countermeasures and improve traffic safety management.

The paper by K. Shaaban explores drivers' perceptions on smartphone applications for driving assistance. The survey was conducted on 421 participants from Qatar with respect to their interests and perceptions in the installation and use of two smartphone applications for real-time route planning and distracted driving prevention. The results can be used by companies and government agencies for development of road safety and efficiency applications, as well as for safety campaign and awareness programs.

The paper by $\mathrm{X}$. Wang et al. addresses active warning systems for highway-rail grade crossings (HRGC) using connected vehicle technologies. To mitigate the risk of collisions at HRGC, a novel active warning system was designed, implemented, and tested based on readily available connected vehicle technologies and devices. The results from simulations and field tests show that the proposed system offers a better estimation of risk probability, which enhances the system effectiveness and reliability, while the warning messages decrease the collision risk compared to the predicted risk probability.

The paper by K. Kurec et al. presents models and simulations of various controlling aspects of car aerodynamic characteristics by actuating movable aerodynamic adds-on on the car body to increase the traction, braking, and lateral force in road conditions requiring such action. The purpose of this work was to extend the safety limits of fast moving cars by controlling the moving aerodynamic elements.

The paper by D. He et al. is on detecting obstacles quickly and accurately during metro operation by using Google's Inception v3 deep convolution neural networks to classify the common facility images from metro tunnels. The developed module provides better quality than other existing methods chosen for comparison while using fewer model parameters 
and reducing computational costs. The use of this approach for metro intelligent monitoring systems could improve the safety of metro operation.

The paper by S. Zheng et al. aims to develop a prediction model for car body vibration acceleration by taking into account railway parameters collected by various sensors and the previous vibration acceleration. The model was based on several training algorithms and neural network structures and was successfully applied to predict the car body vibration acceleration of test datasets on different railway segments. This approach overcomes the complexity and uncertainty of the multiparameters coupling analysis used in traditional models and offers an important instrument to assist the driver in ensuring a smooth operation of the train.

The paper by H.-K. Kim et al. provides a guideline for designing a traffic light recognition system which is useful for advanced driving assistance systems, as well as for autonomous vehicles. The investigation is focused on two main components of the traffic light detection system: the color space of the input video and the network model of deep learning, by considering six color spaces and three types of network models and by comparing the results of all possible combinations on a traffic light dataset.

The paper by K. Lee et al. proposes a novel approach to remotely estimating the heart rate in actual driving environments by using facial images. The driver face region is first detected, and the components related to heart rate are then extracted by using ensemble empirical mode decomposition and analyzed in frequency domain. Based on public human-computer-interface dataset, the method was tested and compared to previous studies. In addition, the method was applied to data obtained from an actual driving situation. This work can be used to prevent accidents due to acute heart rate disease by remotely estimating the heart rate and judicially controlling the vehicle.

The paper by S. Tak et al. investigates the corelation between collision risk and driver behavior by analyzing three surrogate safety measures (SSM) in two car-following scenarios. This study provides solutions to improve the current advanced driver assistance systems. Among three analyzed SSM, the one based on deceleration (DSSM) features similarities to human behavior shows a balanced performance to estimate the collision risks in both deceleration and acceleration phase, thus providing enhanced driver' compliance to the ADAS.

The paper by $\mathrm{S}$. Tian et al. proposes a novel algorithm for predicting bus rollover, providing a faster solution to this problem compared to existing estimation algorithms and thus offering additional time for response. The enhanced method predicts the lateral load transfer ratio (LTR), the most common rollover index, and proves its effectiveness in two standard handling tests ("sine with dwell" and "double lane change").

The paper by J. Masino et al. develops two classifiers of road condition by using mining techniques on data provided by acceleration sensors and gyroscopes installed in vehicles. Due to its modular design, the system can be extended and adapted to multiple and various sensors. A detailed presentation of this system design and capabilities is presented in the article.

In the paper by $\mathrm{S}$. Jaktheerangkoon et al., the authors proposed and tested a blind-corner propagation model for intervehicular communication in order to enhance the standard simulation models used in vehicular ad hoc network. The distance calculation for signals traveling through blind corner is properly adjusted by taking into account IEEE 802.11p blind-corner experiments, and an additional parameter that adjusts the standard model to the degree of the obstruction is introduced. Additional real-life experiments are performed to prove the better performance of the developed model when compared to existing obstacle ones. This result can be used to realistically test intervehicle communication protocols and applications for blind-corner scenario.

Taken together, these 12 papers provide key ICT solutions to various challenges faced by the developers of technologies used for vehicle safety and driver assistance.

\section{Conflicts of Interest}

The guest editors declare that there are no conflicts of interest regarding this special issue.

\section{Acknowledgments}

The guest editors would like to thank the reviewers for the time and efforts devoted to the analysis of the submitted manuscripts, as well as for the comments and suggestions used to improve the quality of the accepted articles.

Mihai Dimian
Luc Chassagne
Petru Andrei
Pu Li 


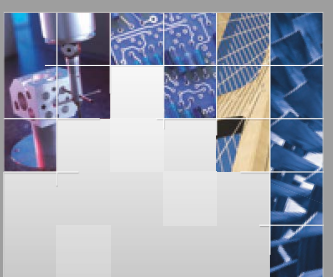

\section{Enfincering}
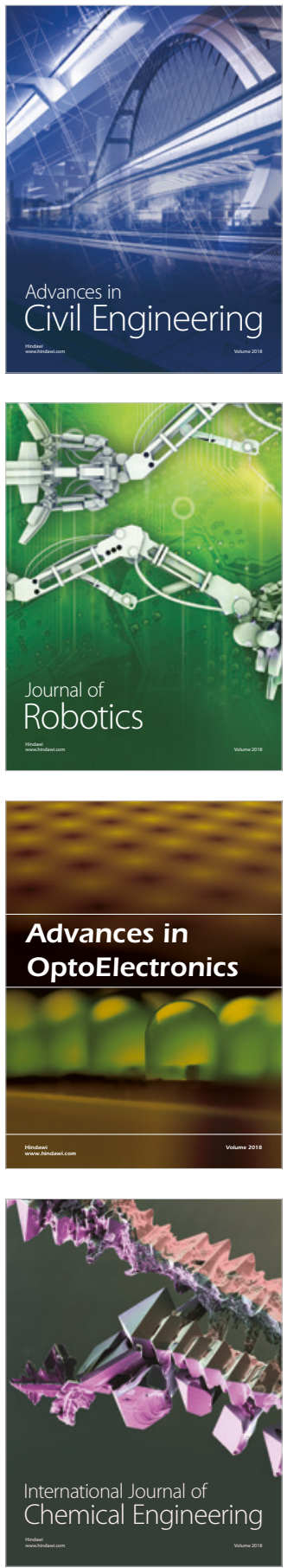

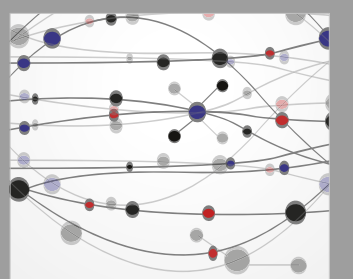

\section{Rotating \\ Machinery}

The Scientific World Journal

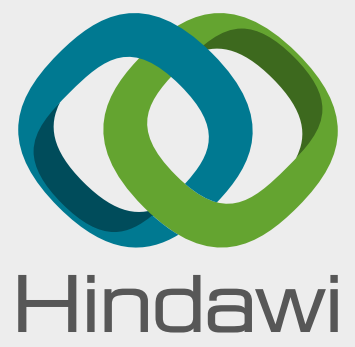

Submit your manuscripts at

www.hindawi.com
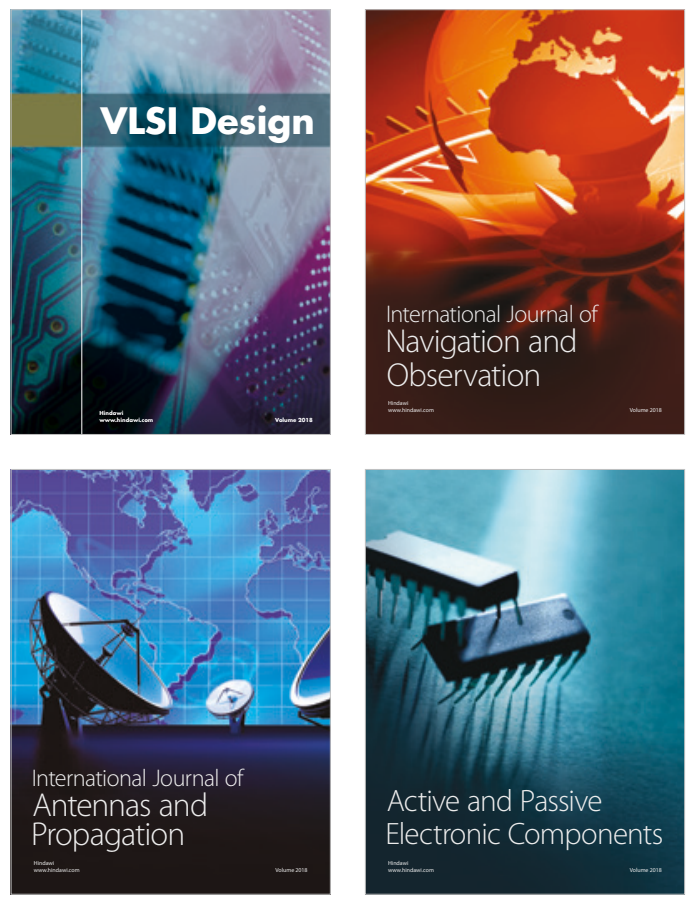
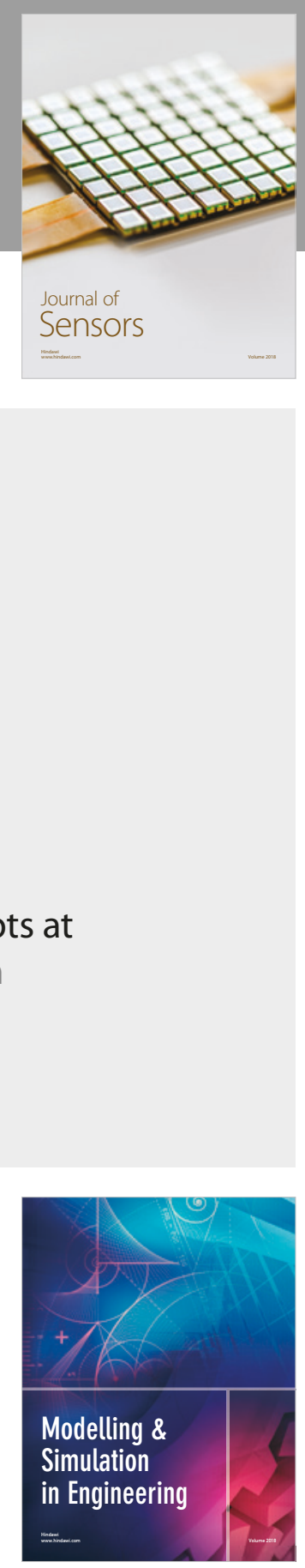

\section{Advances \\ Multimedia}
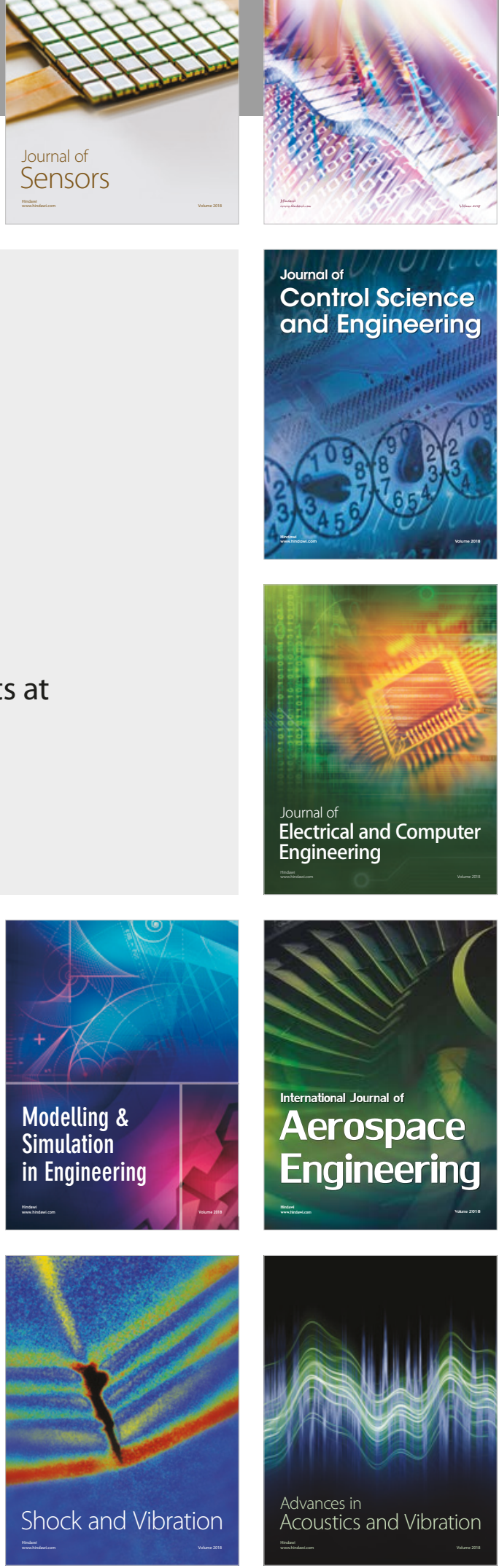